\title{
UTILIZING SUPRA $\alpha$-OPEN SETS TO GENERATE NEW TYPES OF SUPRA COMPACT AND SUPRA LINDELÖF SPACES
}

\author{
Tareq Mohammed Al-shami
}

\begin{abstract}
The purpose of this article is to introduce the concepts of supra $\alpha$-compact (supra $\alpha$-Lindelöf) spaces, almost supra $\alpha$-compact (almost supra $\alpha$-Lindelöf) spaces and mildly supra $\alpha$-compact (mildly supra $\alpha$-Lindelöf) spaces in supra topological spaces utilizing a notion of supra $\alpha$-open sets. Several properties of each concept are established and the relationships among them are provided with the help of interesting examples. A notion of supra $\alpha$-limit points is presented and some properties with supra $\alpha$-compactness are derived.

Keywords:Supra $\alpha$-open set, Supra $\alpha$-compact (Supra $\alpha$-Lindelöf) spaces, Almost (Mildly) supra $\alpha$-compact and almost (mildly) supra $\alpha$-Lindelöf spaces.
\end{abstract}

\section{Introduction}

In 1965, Njastad [13] presented and investigated a notion of $\alpha$-open sets in topological spaces. Mildly compact (Mildly Lindelöf) spaces [15] were introduced in 1974 and almost compact spaces [9] were introduced in 1975 by Staum and Lambrinos, respectively. Mashhour et al.[11] introduced a concept of supra topological spaces and investigated some of its properties in 1983. In 1985, Maheshwari and Thakur [10] initiated a concept of $\alpha$-compact spaces and studied some of its properties. In 2008, Devi et al. [5] generalized a notion of $\alpha$-open sets in topological spaces to supra $\alpha$-open sets in supra topological spaces. Recently, Some notions in supra topological spaces such as supra closure operators, supra compact (supra Lindelöf) spaces and supra regular (supra normal) spaces were introduced and investigated by me [2]. In addition, a new class of generalized open sets called somewhere dense [3] was studied and discussed in detail. It is well known that topological spaces and supra topological spaces have been generalised and studied in many ways (see for example, [1], [4], [7], [8], [16], [17]).

In the present article, we introduce and study the concepts of supra $\alpha$-compact

Received July 31, 2016; accepted February 04, 2017

2010 Mathematics Subject Classification. Primary 54D20; Secondary 54D30; 54A05. 
(supra $\alpha$-Lindelöf) spaces, almost supra $\alpha$-compact (almost supra $\alpha$-Lindelöf) spaces and mildly supra $\alpha$-compact (mildly supra $\alpha$-Lindelöf) spaces in supra topological spaces by using a notion of supra $\alpha$-open sets. The characterizations of these concepts are investigated. Apart from that some illustrative examples are presented to point out relationships among these concepts. A notion of supra $\alpha$-limit points is introduced and some properties with supra $\alpha$-compact spaces are investigated. Several interesting results are derived which may be value of further researches.

\section{Preliminaries}

Definition 2.1. [11] A sub-collection $\mu$ of $2^{X}$ is called a supra topology on $X$ if it satisfies the following two axioms

(i) $X$ belongs to $\mu$.

(ii) $\mu$ is closed under arbitrary union.

Every element of $\mu$ is called a supra open set and its complement is called a supra closed set.

Definition 2.2. [5] A subset $E$ of a supra topological space is called supra $\alpha$-open if $E \subseteq \operatorname{sint}(\operatorname{scl}(\operatorname{sint}(E)))$.

Definition 2.3. [5] Let $(X, \mu)$ be a supra topological space, $(Y, \theta)$ be a topological space and $\rho$ be an associated supra topology with $\theta$. A map $g:(X, \mu) \rightarrow(Y, \theta)$ is called supra $\alpha$-continuous if the inverse image of each open set in $Y$ is a supra $\alpha$-open set in $X$.

Definition 2.4. ([5],[11]) Let $E$ be a subset of a supra topological space $(X, \mu)$. Then

(i) Supra interior of $E$, denoted by $\sin t(E)$, is the union of all supra open sets contained in $E$.

(ii) Supra closure of $E$, denoted by $\operatorname{scl}(E)$, is the intersection of all supra open sets containing $E$.

(iii) Supra $\alpha$-interior of $E$, denoted by saint $(E)$, is the union of all supra $\alpha$-open sets contained in $E$.

(iv) Supra $\alpha$-closure of $E$, denoted by $\operatorname{sacl}(E)$, is the intersection of all supra $\alpha$-open sets containing $E$.

Definition 2.5. [2] A supra topological space $(X, \mu)$ is said to be 
(i) Supra compact (resp. supra Lindelöf) provided that every supra open cover of $X$ has a finite (resp. countable) subcover.

(ii) Almost supra compact (resp. almost supra Lindelöf) provided that every supra open cover of $X$ has a finite (resp. countable) sub-collection the supra closure of whose member cover $X$.

(iii) Mildly supra compact (resp. mildly supra Lindelöf) provided every supra clopen cover of $X$ has a finite (resp. countable) subcover.

Definition 2.6. [14] Let $g$ be a map of $X$ into $Y$. Let $\left\{A_{i}: i \in I\right\}$ and $\left\{B_{i}: i \in I\right\}$ be a collection of subsets of $X$ and $Y$, respectively. Then

(i) $g\left(\bigcup_{i \in I} A_{i}\right)=\bigcup_{i \in I} g\left(A_{i}\right)$.

(ii) $g^{-1}\left(\bigcup_{i \in I} B_{i}\right)=\bigcup_{i \in I} g^{-1}\left(B_{i}\right)$.

Throughout this paper, the set of natural numbers, the set of rational numbers and the set of real numbers are denoted by $\mathcal{N}, \mathcal{Q}$ and $\mathcal{R}$, respectively.

\section{Supra $\alpha$-compact and supra $\alpha$-Lindelöf spaces}

We introduce in this section the concepts of supra $\alpha$-compact and supra $\alpha$-Lindelöf spaces. The relationships between them are studied with the help of examples and the equivalent conditions for each one of them are given.

Definition 3.1. A collection $\left\{G_{i}: i \in I\right\}$ of supra $\alpha$-open sets in a supra topological spaces $(X, \mu)$ is called a supra $\alpha$-open cover of a subset $E$ of $X$ provided that $E \subseteq \bigcup\left\{G_{i}: i \in I\right\}$.

Definition 3.2. A supra topological spaces $(X, \mu)$ is called supra $\alpha$-compact (resp. supra $\alpha$-Lindelöf) provided that every supra $\alpha$-open cover of $X$ has a finite (resp. countable) subcover.

In the following, we give two examples, the first one satisfies the concept of supra $\alpha$-compactness and the second one does not satisfy.

Example 3.1. Let $\mu=\left\{\varnothing, G_{n} \subseteq \mathcal{N}: G_{n}=\{n, n+1, n+2, \ldots: n \in \mathcal{N}\}\right\}$ be a supra topology on $\mathcal{N}$. Since infimum $\left(G_{n}\right)=n$, for any subset $G_{n}$ of $(\mathcal{N}, \mu)$, then $(\mathcal{N}, \mu)$ is supra $\alpha$-compact.

Example 3.2. Let $\mu=\{\varnothing, G \subseteq \mathcal{N}: \exists x \in G$ such that $2 \mid x\}$ be supra topology on $\mathcal{N}$. Now, $\Lambda=\{\{n, n+1\}: n \in \mathcal{N}\}$ is a supra $\alpha$-open cover of $\mathcal{N}$. As $\Lambda$ has not a finite subcover, then $(\mathcal{N}, \mu)$ is not supra $\alpha$-compact. 
Proposition 3.1. Every supra $\alpha$-compact space is supra $\alpha$-Lindelöf.

Proof. It is straightforward.

The converse of the above proposition need not be true as shown in Example 3.2.

Proposition 3.2. Any finite (resp. countable) supra topological space $(X, \mu)$ is supra $\alpha$-compact (resp. supra $\alpha$-Lindelöf).

Proof. (i) If $X$ is finite, then the proof comes immediately from the fact that $P(X)$ is finite

(ii) If $X$ is countable, then the largest cover with respect to the number of its element is $\Lambda=\{\{x\}: x \in X\}$. The countability of $\Lambda$ completes the proof.

Proposition 3.3. A finite (resp. countable) union of supra $\alpha$-compact subsets of a supra topological space $(X, \mu)$ is supra $\alpha$-compact (resp. supra $\alpha$-Lindelöf).

Proof. It is straightforward.

Proposition 3.4. Every supra $\alpha$-compact (resp. supra $\alpha$-Lindelöf) space $(X, \mu)$ is supra compact (resp. supra Lindelöf).

Proof. This is easily obtained from the fact that every supra open set is supra $\alpha$-open.

To see that the converse of the above proposition is not always true, we give the following example.

Example 3.3. Let $\mu=\left\{\varnothing, \mathcal{R}, G_{n} \subseteq \mathcal{R}: G_{n}=\{n, n+1, n+2, \ldots: n \in \mathcal{N}\}\right\}$ be a supra topology on $\mathcal{R}$. Obviously, $(\mathcal{R}, \mu)$ is supra compact. A collection $\Lambda=\left\{\mathcal{Q} \bigcup\{x\}: x \in \mathcal{Q}^{c}\right\}$ is a supra $\alpha$-open cover of $\mathcal{R}$. Since $\Lambda$ has not a countable subcover of $\mathcal{R}$, then $(\mathcal{R}, \mu)$ is not supra $\alpha$-Lindelöf.

Definition 3.3. A subset $E$ of a supra topological space $(X, \mu)$ is said to be supra $\alpha$-compact (resp. supra $\alpha$-Lindelöf) relative to $X$ if every supra $\alpha$-open cover of $E$, reducible to a finite (resp. countable) subcover.

Theorem 3.1. Every supra $\alpha$-closed subset of a supra $\alpha$-compact (resp. supra $\alpha$-Lindelöf) space $(X, \mu)$ is supra $\alpha$-compact (resp. supra $\alpha$-Lindelöf).

Proof. Let $F$ be a supra $\alpha$-closed subset of $X$ and $\left\{G_{i}: i \in I\right\}$ be a supra $\alpha$-open cover of $F$. Then $F^{c}$ is supra $\alpha$-open and $F \subseteq \bigcup_{i \in I} G_{i}$. Therefore $X=\bigcup_{i \in I} G_{i} \bigcup F^{c}$. Since $X$ is supra $\alpha$-compact, then $X=\bigcup_{i=1}^{i=n} G_{i} \cup F^{c}$. Thus $F \subseteq \bigcup_{i=1}^{i=n} G_{i}$. Hence $F$ is supra $\alpha$-compact.

One can prove theorem similarly in case of supra $\alpha$-Lindelöf spaces. 
Definition 3.4. [14] A collection $\left\{G_{i}: i \in I\right\}$ of sets is said to have the finite intersection property if every finite sub-collection of $\left\{G_{i}: i \in I\right\}$ has a non-empty intersection.

Theorem 3.2. A supra topological space $(X, \mu)$ is supra $\alpha$-compact if and only if every collection of supra $\alpha$-closed subsets of $X$, satisfying the finite intersection property, has, itself, a non-empty intersection.

Proof. Necessity: Let $\Lambda=\left\{F_{i}: i \in I\right\}$ be a collection of supra $\alpha$-closed subsets of $X$ which has the finite intersection property. Assume that $\bigcap_{i \in I} F_{i}=\varnothing$. Then $X=\bigcup_{i \in I} F_{i}^{c}$. Since $X$ is supra $\alpha$-compact, then $X=\bigcup_{i=1}^{i=n} F_{i}^{c}$. Therefore $\bigcap_{i=1}^{i=n} F_{i}=\varnothing$. But this contradicts that $\Lambda$ has a finite intersection property. Thus $\Lambda$ has a nonempty intersection.

Sufficiency: Let $\Omega=\left\{G_{i}: i \in I\right\}$ be a supra $\alpha$-open cover of $X$. Suppose $\Omega$ has no finite subcover. Then $X \backslash \bigcup_{i=1}^{i=n} G_{i} \neq \varnothing$, for any $n \in \mathcal{N}$. Now, $\bigcap_{i=1}^{i=n} G_{i}^{c} \neq \varnothing$ implies that $\left\{G_{i}^{c}: i \in I\right\}$ is a collection of supra $\alpha$-closed subsets of $X$ which has the finite intersection property. Therefore $\bigcap_{i \in I} G_{i}^{c} \neq \varnothing$. Thus $X \neq \bigcup_{i \in I} G_{i}$. But this contradicts that $\Omega$ is a supra $\alpha$-open cover of $X$. Hence $(X, \mu)$ is supra $\alpha$-compact.

Definition 3.5. A collection $\left\{G_{i}: i \in I\right\}$ of sets is said to have the countable intersection property if every countable sub-collection of $\left\{G_{i}: i \in I\right\}$ has a nonempty intersection.

The proof of the following theorem is similar of that Theorem 3.2.

Theorem 3.3. A supra topological space $(X, \mu)$ is supra $\alpha$-Lindelöf if and only if every collection of supra $\alpha$-closed subsets of $X$, satisfying the countable intersection property, has, itself, a non-empty intersection.

Theorem 3.4. If $A$ is a supra $\alpha$-compact (resp. supra $\alpha$-Lindelöf) subset of $X$ and $B$ is a supra $\alpha$-closed subset of $X$, then $A \cap B$ is supra $\alpha$-compact (resp. supra $\alpha$-Lindelöf).

Proof. We prove theorem in case of supra $\alpha$-Lindelöf spaces and the other case is proven similarly.

Let $\Lambda=\left\{G_{i}: i \in I\right\}$ be a supra $\alpha$-open cover of a set $A \cap B$. Then $A \subseteq \bigcup_{i \in I} G_{i} \bigcup B^{c}$.

Since $A$ is supra $\alpha$-Lindelöf, then there exists a countable set $S$ such that $A \subseteq$ $\bigcup_{i \in S} G_{i} \cup B^{c}$. Therefore $A \cap B \subseteq \bigcup_{i \in S} G_{i}$. Thus the set $A \cap B$ is supra $\alpha$-Lindelöf.

Theorem 3.5. The supra $\alpha$-continuous image of a supra $\alpha$-compact (resp. supra $\alpha$-Lindelöf) set is compact (resp. Lindelöf). 
Proof. Let $g: X \rightarrow Y$ be a supra $\alpha$-continuous map and let $A$ be a supra $\alpha$ compact subset of $X$. Suppose that $\left\{G_{i}: i \in I\right\}$ is an open cover of $g(A)$. Now, $g(A) \subseteq \bigcup_{i \in I} G_{i}$ implies that $A \subseteq \bigcup_{i \in I} g^{-1}\left(G_{i}\right)$. Since $g$ is supra $\alpha$-continuous, then $g^{-1}\left(G_{i}\right)$ is a supra $\alpha$-open set, for all $i \in I$. By hypotheses, $A$ is supra $\alpha$-compact, then $g(A) \subseteq \bigcup_{i=1}^{i=n} G_{i}$. Hence $g(A)$ is compact.

Similarly, one can prove theorem in case of supra $\alpha$-Lindelöf spaces.

Definition 3.6. Let $A$ be a subset of $(X, \mu)$ and $x \in X$. A point $x$ is said to be supra $\alpha$-limit point of $A$ provided that every supra $\alpha$-open set containing $x$ contains at least one point of $A$ different than $x$.

The set of all supra $\alpha$-limit points of $A$ is denoted by $A^{s \alpha l}$.

Theorem 3.6. Let $A$ be a subset of $(X, \mu)$. Then $A$ is supra $\alpha$-closed if and only if $A^{s \alpha l} \subseteq A$.

Proof. Necessity: Assume that $A$ is supra $\alpha$-closed and $x \notin A$. Then $x \in A^{c}$. As $A^{c}$ is supra $\alpha$-open and $A^{c} \bigcap A=\emptyset$, then $x \notin A^{s \alpha l}$. Therefore $A^{s \alpha l} \subseteq A$.

Sufficiency: Let $x \in A^{c}$ and $A^{s \alpha l} \subseteq A$. Then $x \notin A^{s \alpha l}$. Therefore there exists a supra $\alpha$-open set $G_{x}$ such that $G_{x} \backslash\{x\} \cap A=\varnothing$. As $x \in A^{c}$, then $G_{x} \bigcap A=\varnothing$. Now, $G_{x} \subseteq A^{c}$. Therefore $A^{c}=\bigcup\left\{G_{x}: x \in A^{c}\right\}$. Thus $A^{c}$ is a supra $\alpha$-open set. Hence $A$ is supra $\alpha$-closed.

Corollary 3.1. Let $A$ be a subset of supra $\alpha$-compact (resp. supra $\alpha$-Lindelöf) space. Then $A^{\text {sal }}$ is supra $\alpha$-compact (resp. supra $\alpha$-Lindelöf).

Theorem 3.7. Every infinite subset of a supra $\alpha$-compact space has a supra $\alpha$ limit point.

Proof. Let $A$ be an infinite subset of a supra $\alpha$-compact space $(X, \mu)$. Suppose that $A$ has not a supra $\alpha$-limit point. Then for each $x_{i} \in X$, there exists a supra $\alpha$-open set $G_{x_{i}}$ such that $G_{x_{i}} \cap A \backslash\left\{x_{i}\right\}=\varnothing$. Now, the collection $\Lambda=\left\{G_{x_{i}}: x_{i} \in X\right\}$ forms a supra $\alpha$-open cover of $X$. As $X$ is supra $\alpha$-compact, then $X=\bigcup_{i=1}^{i=n} G_{x_{i}}$. Therefore $X$ has at most $n$ points of $A$. This implies that $A$ is finite. But this contradicts that $A$ is infinite. Thus $A$ has a supra $\alpha$-limit point.

\section{Almost supra $\alpha$-compact and almost supra $\alpha$-Lindelöf spaces}

This section is devoted to presenting some features of almost supra $\alpha$-compact and almost supra $\alpha$-Lindelöf spaces. Some examples are given to illustrate the main results.

Definition 4.1. A supra topological spaces $(X, \mu)$ is called almost supra $\alpha$-compact (resp. almost supra $\alpha$-Lindelöf) provided that every supra $\alpha$-open cover of $X$ has a finite (resp. countable) sub-collection the supra $\alpha$-closure of whose member cover $X$. 
In the following, we give two examples, the first one satisfies the concept of almost supra $\alpha$-compactness and the second one does not satisfy.

Example 4.1. Let $\mu=\left\{\varnothing, G_{n}=\{1,2, \ldots, 2 n+1\}\right.$ or $\left.G_{n}=\{1,2, \ldots, 3 n+1\}: n \in \mathcal{N}\right\}$ be a supra topology on $\mathcal{N}$. Any non-empty supra open subset of $(\mathcal{N}, \mu)$ is dense. It is well known that every non-empty supra $\alpha$-open set containing a non-empty supra open set and then every non-empty supra $\alpha$-open subset of $(\mathcal{N}, \mu)$ is dense. Thus $(\mathcal{N}, \mu)$ is almost supra $\alpha$-compact. On the other hand, let $\Lambda=\{\{1,2,3, x\}: x \in \mathcal{N}$ and $x>3\}$ be a supra $\alpha$-open cover of $\mathcal{N}$. Since $\Lambda$ has not finite subcover, then $(\mathcal{N}, \mu)$ is not supra $\alpha$-compact.

Example 4.2. Let $\mu=\{\varnothing, G \subseteq \mathcal{Q}$ such that $G$ containing at least two elements $\}$ be a supra topology on $\mathcal{Q}$ and let $\Lambda$ be the collection of all sets which consist of only two elements. Then $(\mathcal{Q}, \mu)$ is not almost supra $\alpha$-compact.

Proposition 4.1. Every almost supra $\alpha$-compact space is almost supra $\alpha$-Lindelöf.

Proof. It is straightforward.

The converse of the above proposition need not be true as illustrated in Example 4.2 .

Proposition 4.2. Every supra $\alpha$-compact (resp. supra $\alpha$-Lindelöf) space $(X, \mu)$ is almost supra $\alpha$-compact (resp. almost supra $\alpha$-Lindelöf).

Proof. It comes immediately from the fact that every set is contained in its supra $\alpha$-closure.

The converse of the above theorem does not hold as shown in the following example.

Example 4.3. Let $\mu=\{\varnothing, G \subseteq \mathcal{R}$ such that $1 \in G\}$ be a supra topology on $\mathcal{R}$. Since $\operatorname{s\alpha cl}(\{1\})=\mathcal{R}$, then $(\mathcal{R}, \mu)$ is almost supra $\alpha$-compact. On the other hand, let $\Lambda=$ $\{\{1, x\}: x \in \mathcal{R}\}$ be a collection of supra $\alpha$-open sets. Obviously, $\Lambda$ has not a countable cover of $\mathcal{R}$. Hence $(\mathcal{R}, \mu)$ is not supra $\alpha$-Lindelöf.

Next two propositions with immediate proof and then omitted.

Proposition 4.3. Every finite (resp. countable) supra topological space $(X, \mu)$ is almost supra $\alpha$-compact (resp. almost supra $\alpha$-Lindelöf).

Proposition 4.4. A finite (resp. countable) union of almost supra $\alpha$-compact subsets of a supra topological space $(X, \mu)$ is almost supra $\alpha$-compact (resp. supra $\alpha$-Lindelöf).

Definition 4.2. A subset $E$ of a supra topological space $(X, \mu)$ is called supra $\alpha$-clopen provided that $E$ is supra $\alpha$-open and supra $\alpha$-closed.

Remark 4.1. A subset $E$ of a supra topological space $(X, \mu)$ is supra $\alpha$-clopen if and only if $\operatorname{scl}(\operatorname{sint}(\operatorname{scl}(E))) \subseteq E \subseteq \operatorname{sint}(\operatorname{scl}(\operatorname{sint}(E)))$. 
Theorem 4.1. If $F$ is a supra $\alpha$-clopen subset of an almost supra $\alpha$-compact (resp. almost supra $\alpha$-Lindelöf) space $(X, \mu)$, then $F$ is almost supra $\alpha$-compact (resp. almost supra $\alpha$-Lindelöf).

Proof. Let $F$ be a supra $\alpha$-clopen subset of $X$ and let $\left\{G_{i}: i \in I\right\}$ be a supra $\alpha$-open cover of $F$. Then $F^{c}$ is supra $\alpha$-clopen and $F \subseteq \bigcup_{i \in I} G_{i}$. Therefore $X=$ $\bigcup_{i \in I} G_{i} \bigcup F^{c}$. Since $X$ is almost supra $\alpha$-compact, then $X=\bigcup_{i=1}^{i=n} \operatorname{socl}\left(G_{i}\right) \bigcup F^{c}$. Thus $F \subseteq \bigcup_{i=1}^{i=n} \operatorname{sicl}\left(G_{i}\right)$. Hence $F$ is almost supra $\alpha$-compact.

The proof for supra $\alpha$-Lindelö spaces is similar.

Theorem 4.2. If $A$ is an almost supra $\alpha$-compact (resp. almost supra $\alpha$-Lindelöf) subset of $X$ and $B$ is a supra $\alpha$-clopen subset of $X$, then $A \cap B$ is almost supra $\alpha$ compact (resp. almost supra $\alpha$-Lindelöf).

Proof. Let $\Lambda=\left\{G_{i}: i \in I\right\}$ be a supra $\alpha$-open cover of $A \bigcap B$. Then $A \subseteq$ $\bigcup_{i \in I} G_{i} \bigcup B^{c}$. Since $A$ is an almost supra $\alpha$-compact set, then $A \subseteq \bigcup_{i=1}^{i=n} \operatorname{socl}\left(G_{i}\right) \bigcup B^{c}$. Therefore $A \cap B \subseteq \bigcup_{i=1}^{i=n} \operatorname{socl}\left(G_{i}\right)$. Thus $A \cap B$ is almost supra $\alpha$-compact. The proof for almost supra $\alpha$-Lindelöf spaces is similar.

Theorem 4.3. The supra $\alpha$-continuous image of an almost supra $\alpha$-compact (resp. almost supra $\alpha$-Lindelöf) set is almost compact (resp. almost Lindelöf).

Proof. Let $g: X \rightarrow Y$ be a supra $\alpha$-continuous map and let $A$ be an almost supra $\alpha$-compact subset of $X$. Suppose that $\left\{G_{i}: i \in I\right\}$ be an open cover of $g(A)$. Now, $g(A) \subseteq \bigcup_{i \in I} G_{i}$ implies that $A \subseteq \bigcup_{i \in I} g^{-1}\left(G_{i}\right)$. Since $g$ is supra $\alpha$-continuous, then $g^{-1}\left(G_{i}\right)$ is a supra $\alpha$-open set, for each $i \in I$. By hypotheses, $A$ is almost supra $\alpha$-compact, then $A \subseteq \bigcup_{i=1}^{i=n} \operatorname{socl}\left(g^{-1}\left(G_{i}\right)\right)$. Since $g$ is supra $\alpha$-continuous, then $\operatorname{s\alpha cl}\left(g^{-1}\left(G_{i}\right)\right) \subseteq g^{-1}\left(c l\left(G_{i}\right)\right)$. Therefore $g(A) \subseteq \bigcup_{i=1}^{i=n} g\left(\left(g^{-1}\left(c l\left(G_{i}\right)\right)\right)\right) \subseteq \bigcup_{i=1}^{i=n} c l\left(G_{i}\right)$. Hence $g(A)$ is almost compact.

The proof for almost supra $\alpha$-Lindelöf spaces is similar.

Theorem 4.4. If every collection of supra $\alpha$-closed subsets of a supra topological space $(X, \mu)$, satisfying the finite intersection property, has, itself, a non-empty intersection, then $X$ is almost supra $\alpha$-compact.

Proof. Let $\Lambda=\left\{G_{i}: i \in I\right\}$ be a supra $\alpha$-open cover of $X$. Suppose $\left\{G_{i}: i \in I\right\}$ has no finite sub-collection which is the supra $\alpha$-closure of whose member cover 
$X$. Then $X \backslash \bigcup_{i=1}^{i=n} \operatorname{sacl}\left(G_{i}\right) \neq \varnothing$, for any $n \in \mathcal{N}$. Therefore $X \backslash \bigcup_{i=1}^{i=n} G_{i} \neq \varnothing$. Now, $\bigcap_{i=1}^{i=n} G_{i}^{c} \neq \varnothing$ and this implies that $\left\{G_{i}^{c}: i \in I\right\}$ is a collection of supra $\alpha$-closed subsets of $X$ which has the finite intersection property. Thus $\bigcap_{i \in I} G_{i}^{c} \neq \varnothing$ and this implies that $X \neq \bigcup_{i \in I} G_{i}$. But this contradicts that $\Lambda$ is a supra $\alpha$-open cover of $X$. Hence $X$ is almost supra $\alpha$-compact.

The converse of the above theorem is not always true as shown in the following: The supra topological space $(\mathcal{N}, \mu)$ which is defined in Example 4.1 is almost supra $\alpha$-compact. A collection of supra $\alpha$-closed subsets $\left\{A_{n}: A_{n}=\{2 n+2,2 n+3, \ldots\}\right.$ and $n \in \mathcal{N}\}$ of $(\mathcal{N}, \mu)$ has a finite intersection property, whereas $\bigcap_{i=1}^{\infty} A_{n}=\varnothing$.

\section{Mildly supra $\alpha$-compact and mildly supra $\alpha$-Lindelöf spaces}

In this section, we introduce the concepts of mildly supra $\alpha$-compact and mildly supra $\alpha$-Lindelöf spaces and illustrate its relationship with the previous four supra compact spaces.

Definition 5.1. A supra topological space $(X, \mu)$ is called mildly supra $\alpha$-compact (resp. mildly supra $\alpha$-Lindelöf) provided that every supra $\alpha$-clopen cover of $X$ has a finite (resp. countable) subcover.

Proposition 5.1. Every mildly supra $\alpha$-compact is mildly supra $\alpha$-compact space is mildly supra $\alpha$-Lindelöf.

Proof. Straightforward.

If we replace $\mathcal{R}$ by $\mathcal{Q}$ in Example 4.2, then $\Lambda=\{\{1, x, y\}: x \neq y \neq 1 \in \mathcal{Q}\}$ is a supra $\alpha$-clopen cover of $\mathcal{Q}$. Obviously, $\Lambda$ has a countable cover of $\mathcal{Q}$, whereas it has not a finite subcover. Hence the converse of the above proposition need not be true in general.

Theorem 5.1. Every almost supra $\alpha$-compact (resp. almost supra $\alpha$-Lindelöf) space $(X, \mu)$ is mildly supra $\alpha$-compact (resp. mildly supra $\alpha$-Lindelöf).

Proof. Let $\Lambda=\left\{H_{i}: i \in I\right\}$ be a supra $\alpha$-clopen cover of $(X, \mu)$. Since $(X, \mu)$ is almost supra $\alpha$-compact, then $X=\bigcup_{i=1}^{i=n} \operatorname{socl}\left(H_{i}\right)$ and since $H_{i}$ is a supra $\alpha$-clopen set, then $\operatorname{s\alpha cl}\left(H_{i}\right)=H_{i}$. Thus $(X, \mu)$ is mildly supra $\alpha$-compact.

The proof for mildly supra $\alpha$-Lindelöf spaces is similar. 
Corollary 5.1. Every supra $\alpha$-compact (resp. supra $\alpha$-Lindelöf) space is mildly supra $\alpha$-compact (mildly supra $\alpha$-Lindelöf).

The following interesting example points out that the converse of the above theorem is not always true.

Example 5.1. Let $\mu=\{\varnothing, G \subseteq \mathcal{R}$ such that $G=(a, \infty)$ or $G=(-\infty, b)$ or their union $: a, b \in \mathcal{R}\}$ be a supra topology on $\mathcal{R}$. In the following we show that every supra $\alpha$-open subsets of $(\mathcal{R}, \mu)$ is supra open and then the only supra $\alpha$-clopen sets are $\varnothing$ and $\mathcal{R}$. Suppose that $A$ is not a supra open subset of $(\mathcal{R}, \mu)$. Then we have the following five possible cases.

1. $A=[a, \infty)$ or $A=(-\infty, a] \Rightarrow a \notin \operatorname{sint}(\operatorname{scl}(\operatorname{sint}(A)))$. Therefore $A$ is not supra $\alpha$-open.

2. $A=[a, \infty) \bigcup(-\infty, b)$ or $A=(-\infty, a] \bigcup(b, \infty)$ such that $a \neq b \Rightarrow a \notin \operatorname{sint}(\operatorname{scl}(\operatorname{sint}(A)))$. Therefore $A$ is not supra $\alpha$-open.

3. $A=(a, b)$ or $A=[a, b]$ or their union $\Rightarrow \operatorname{sint}(A)=\varnothing$. Therefore $A$ is not supra $\alpha$-open.

4. $A=\left\{x_{i}: i \in I\right\}$ such that $A$ is not interval $\Rightarrow A$ is not supra $\alpha$-open.

5. $A$ is written as a union of any number of the above cases $\Rightarrow A$ is not supra $\alpha$-open.

From the above discussion, we can conclude that $A$ is a supra $\alpha$-open subset of $(\mathcal{R}, \mu)$ if and only if it is supra open. Thus the only supra $\alpha$-clopen subsets of $(\mathcal{R}, \mu)$ are $\mathcal{R}$ and $\varnothing$. Hence $(\mathcal{R}, \mu)$ is mildly supra $\alpha$-compact.

On the other hand, there is not a finite subcover that the supra $\alpha$-closure of whose member cover $\mathcal{R}$, for a supra $\alpha$-open cover $\{(-\infty, b): b \in \mathcal{R}\}$ of $\mathcal{R}$. Hence $(\mathcal{R}, \mu)$ is not almost supra $\alpha$-compact.

Proposition 5.2. Any finite (resp. countable) supra topological space is mildly supra $\alpha$-compact (resp. mildly supra $\alpha$-Lindelöf).

Proof. This is easily obtained from Corollary 5.1 and Proposition 3.2.

Proposition 5.3. A finite (resp. countable) union of mildly supra $\alpha$-compact subsets of a supra topological space is mildly supra $\alpha$-compact (resp. mildly supra $\alpha$ Lindelöf).

Proof. This is easily obtained from Corollary 5.1 and Proposition 3.3.

Theorem 5.2. Every supra $\alpha$-clopen subset $F$ of a mildly supra $\alpha$-compact (resp. mildly supra $\alpha$-Lindelöf) space $(X, \mu)$ is mildly supra $\alpha$-compact (resp. mildly supra $\alpha$-Lindelöf).

Proof. Let $F$ be a supra $\alpha$-clopen subset of $X$ and let $\left\{G_{i}: i \in I\right\}$ be a supra $\alpha$-clopen cover of $F$. Then $F^{c}$ is supra $\alpha$-clopen and $F \subseteq \bigcup_{i \in I} G_{i}$. Therefore $X=$ 
$\bigcup_{i \in I} G_{i} \bigcup F^{c}$. Since $X$ is mildly supra $\alpha$-compact, then $X=\bigcup_{i=1}^{i=n} G_{i} \bigcup F^{c}$. Thus $F \subseteq \bigcup_{i=1}^{i=n} G_{i}$. Hence $F$ is mildly supra $\alpha$-compact.

The proof for mildly supra $\alpha$-Lindelöf spaces is similar.

Theorem 5.3. If $A$ is a mildly supra $\alpha$-compact (resp. mildly supra $\alpha$-Lindelöf) subset of $(X, \mu)$ and $B$ is a supra $\alpha$-clopen subset of $(X, \mu)$, then $A \cap B$ is mildly supra $\alpha$-compact (resp. mildly supra $\alpha$-Lindelöf).

Proof. Let $\Lambda=\left\{G_{i}: i \in I\right\}$ be a supra $\alpha$-clopen cover of $A \cap B$. Then $A \subseteq$ $\bigcup_{i \in I} G_{i} \cup B^{c}$. Since $A$ is mildly supra $\alpha$-compact, then $A \subseteq \bigcup_{i=1}^{i=n} G_{i} \bigcup B^{c}$. Therefore $A \cap B \subseteq \bigcup_{i=1}^{i=n} G_{i}$. Thus $A \cap B$ is mildly supra $\alpha$-compact.

The proof for mildly supra $\alpha$-Lindelöf spaces is similar.

Theorem 5.4. The supra $\alpha$-continuous image of a mildly supra $\alpha$-compact (resp. mildly supra $\alpha$-Lindelöf) set is compact (resp. Lindelöf).

Proof. The proof is similar of that Theorem 3.5.

Remark 5.1. A collection of all supra $\alpha$-open subsets of a supra topological space $(X, \mu)$, denoted by $\mu^{s \alpha}$, forms a supra topology on $X$, .

Theorem 5.5. Consider $\left(X, \mu^{s \alpha}\right)$ has a supra base consists of supra $\alpha$-clopen sets. Then $(X, \mu)$ is supra $\alpha$-compact if and only if it is mildly supra $\alpha$-compact.

Proof. The necessary condition is clear.

To prove the sufficient condition, let $\Lambda$ be a supra $\alpha$-open cover of a mildly supra $\alpha$-compact space $(X, \mu)$. Since $\left(X, \mu^{s \alpha}\right)$ has a supra base consists of supra $\alpha$-clopen sets, then every covering of $X$ by members of this supra base has finite subcover. This completes the proof.

\section{Conclusion}

In the present work, we have studied the concepts of supra $\alpha$-compact (supra $\alpha$ Lindelöf) spaces, almost (mildly) supra $\alpha$-compact spaces and almost (mildly) supra $\alpha$-Lindelöf spaces in supra topological spaces depending on a notion of supra $\alpha$-open sets. Also, we have investigated the main properties of these concepts and have illustrated the relationships among them. In a next study, we use a notion of supra $R$-open sets [6] to generalize some of these concepts. We hope that the results obtained in this article will help research teams promote further study in supra topological spaces to carry out a general frame work for the practical applications. 


\section{R E F E R E N C E S}

1. M. Abo-elhamaye And T. M. Al-Shami, Supra homeomorphism in supra topological ordered spaces, Facta Universitatis, Series: Mathematics and Informatics, 31 (5) (2016) 1091-1106.

2. T. M. AL-Shami, Some results related to supra topological spaces, Journal of Advanced Studies in Topology, 7 (4) (2016) 283-294.

3. T. M. Al-Shami, Somewhere dense sets and $S T_{1}$-spaces, Punjab University Journal of Mathematics, 49 (2) (2017) Accepted.

4. A. CsÁszÁr, Generalized topology, generalized continuity, Acta Mathematica Hungarica, 96 (2002) 351-357.

5. R. Dev, S. Sampathkumar And M. Caldas, On $\alpha$-open sets and s $\alpha$-continuous maps, General Mathematics, 16 (2008) 77-84.

6. M. E. El-Shafei, M. Abo-elhamayel and T. M. Al-Shami, On supra R-open sets and some applications on topological spaces, Journal of Progressive Research in Mathematics, 8 (2) (2016) 1237- 1248.

7. M. E. El-Shafei, M. Abo-elhamayel and T. M. Al-Shami, Strong separation axioms in supra topological ordered spaces, Mathematical Sciences Letters, 6 (3) (2017) Accepted.

8. M. E. El-Shafei, M. Abo-elhamayel and T. M. Al-shami, Supra $R$ homeomorphism in supra topological ordered spaces, Submitted.

9. P. H. Lambrinos, On almost compact and nearly compact spaces, Rendiconti del Circolo Matematico di Palermo, 24 (1975) 14-18.

10. S. N. Maheshwari And S. S. Thakur, On $\alpha$-compact spaces, Bullton Institution of Mathematics and Academic Science, 13 (1985) 341-347.

11. A. S. Mashhour, A. A. Allam, F. S. Mahmoud and F. H. Kheder, On supra topological spaces, Indian Journal of Pure and Applied Mathematics, 14 (1983) 502-510.

12. L. NachBin, Topology and ordered, D. Van Nostrand Inc. Princeton, New Jersey, 1965.

13. O. NJASTAD, On some classes of nearly open sets, Pasific Journal of Mathematics, 15 (1965) 961-970.

14. M. D. Rashinghania And R. S. Aggarwal, Topology for post graduate of indian university, S. Chand and Company Ltd, New Delhi, (1987).

15. R. Staum, The algebra of bounded continuous functions into nonarchimedean field, Pasific Journal of Mathematics, 50 (1974) 169-185.

16. M. ShaBiR AND M. NAZ, On soft topological spaces, Computers and Mathematics with Applications, 61 (2011) 1786-1799.

17. A. N. TAS AND O. B. OZBAKIR, On some mixed types of continuity on generalized neighborhood systems, Journal of Advanced Studies in Topology, 5 (2014), 32-43.

T. M. Al-shami

Department of Mathematics

Sana'a University - Sana'a - Yemen

tareqalshami83@gmail.com 